\title{
Postoperative hyperglycemia in nondiabetic patients after gastric surgery for cancer: perioperative outcomes
}

\author{
Claudio Fiorillo $^{1}$ - Fausto Rosa ${ }^{1}$ - Giuseppe Quero $^{1} \cdot$ Roberta Menghi $^{1}$. \\ Giovanni Battista Doglietto ${ }^{1} \cdot$ Sergio Alfieri $^{1}$
}

Received: 8 December 2015/ Accepted: 2 June 2016/Published online: 23 June 2016

(c) The International Gastric Cancer Association and The Japanese Gastric Cancer Association 2016

\begin{abstract}
Background Hyperglycemia (HG) is widely known to be associated with increased postoperative complications after colorectal surgery. Very few data on the effects of HG on patients after gastric surgery for cancer are reported in literature. The aim of this study was to evaluate the effects of postoperative $\mathrm{HG}$ in non-diabetic patients undergoing gastrectomy for cancer.

Methods One hundred and ninety-three consecutive gastrectomies for cancer performed between January 2010 and December 2015 were considered. Diabetic patients, and those undergoing pancreatic resections were excluded. Postoperative blood glucose levels were monitored in the first $72 \mathrm{~h}$ after surgery. Postoperative complications, mortality, and postoperative course were analyzed in patients who experienced postoperative HG (blood glucose level; BGL $>125 \mathrm{mg} / \mathrm{dl}$ ) compared with euglycemic patients (BGL $\leq 125 \mathrm{mg} / \mathrm{dl}$ ). Differences between mild HG (BGL between 125 and $200 \mathrm{mg} / \mathrm{dl}$ ) and severe HG (BGL $\geq 200 \mathrm{mg} / \mathrm{dl}$ ) were also analyzed.

Results Ninety-six patients (55.5\%) experienced postoperative HG. In 11 patients (6.4\%), a severe postoperative HG was found. Postoperative BGL $>200 \mathrm{mg} / \mathrm{dl}$ was related to worse outcomes than those experienced by euglycemic patients (and even than patients who experienced mild postoperative HG). The postoperative complications rate was $24.8 \%$ (43 patients out of 173), but significantly higher in patients with postoperative severe
\end{abstract}

Claudio Fiorillo

claudio.fiorillo@hotmail.it

1 Department of Digestive Surgery, “A. Gemelli” Hospital, Catholic University, Largo A. Gemelli 8, Rome, Italy
HG compared to mild HG and normoglycemic patients (63.6, 30.6, and $13 \%$, respectively, $p<0.001$ ).

Conclusion Poor postoperative glycemic control seems to be related to worse postoperative outcomes even in patients undergoing elective gastric surgery for cancer.

Keywords Gastric cancer · Hyperglycemia · Perioperative complications

\section{Introduction}

Postoperative (HG) is widely known to be associated with increased complications after cardiac [1, 2], neuro [3], transplant [4], pancreatic [5], and colorectal surgery [6] and in critically ill patients $[7,8]$.

Uncontrolled $\mathrm{HG}$ has recently been linked to an increased rate of surgical site infection after colorectal and general surgery considering both diabetic and non-diabetic patients [6, 9-12].

A recent study by Kiran et al. [13] analyzed the correlation between uncontrolled postoperative HG within $72 \mathrm{~h}$ from colorectal surgery and postoperative mortality, and infectious and noninfectious complications. The authors demonstrated that even a single postoperative elevated glucose value was adversely associated with morbidity and mortality. Nevertheless, postoperative glycemic control in general surgery is usually overlooked. As reported by Levetan et al. [14], only $59 \%$ of general surgery hospitalized patients underwent glucose monitoring during the postoperative period, and only $54 \%$ of them received insulin therapy. In cardiac surgery, a better glucose control using continuous insulin infusion has demonstrated a significant reduction in surgical site infections and in hospital mortality rates associated with diabetes $[15,16]$. As 
demonstrated by all these studies, HG generated as a physical response to severe stress after major surgery significantly influences postoperative outcomes in both diabetic and nondiabetic surgery.

The aim of this study was to evaluate whether perioperative HG is associated with higher rates of morbidity and mortality in nondiabetic patients undergoing gastric surgery for cancer.

\section{Methods}

A retrospective study was conducted including all patients undergoing surgery for gastric cancer between January 2010 and December 2015 at the Digestive Surgery Unit, “A. Gemelli" Hospital, Rome, identified from an institutional review board-approved database of a tertiary referral center for gastric cancer [17].

All patients undergoing gastric surgery operations for gastric cancer with curative intent were included for analysis.

Diabetic patients (defined as presence of a known diagnosis of diabetes mellitus or HbAlc value greater than $6.5 \%$ ) and patients who underwent exploratory laparotomy without gastric resection were excluded from the study. All the others were analyzed according to their blood glucose value from the first postoperative day to the third postoperative day and divided into the subgroups "normoglycemia", "mild hyperglycemia" and "severe hyperglycemia".

In all cases, prepared infusions of saline and carbohydrates (Isolyte ${ }^{\circledR}$ Baxter $2000 \mathrm{ml} /$ day; glucose $50.0 \mathrm{~g} / \mathrm{l}-$ $200 \mathrm{kcal} / \mathrm{l})$ and saline $(1000 \mathrm{ml} /$ day or Ringer lactate $1000 \mathrm{ml} /$ day) were used in the first $72 \mathrm{~h}$ after surgery. No patient received parenteral nutrition through the use of preparations at higher carbohydrate content or with greater caloric content; no patient was fed through enteral nutrition; no patient resumed oral feeding before the fourth postoperative day. All patients were subjected to glycemic controls of serial blood glucose values using peripheral venous blood sampling and through the use of finger-stick every $4 \mathrm{~h}$, and according to the highest recorded glycemic value, were divided into three groups (Normoglycemic patients, mild and severe HG patients).

"Normoglycemia" was defined as initial postoperative glucose value of $125 \mathrm{mg} / \mathrm{dl}$ or less, "mild HG" as blood glucose value between 126 and $199 \mathrm{mg} / \mathrm{dl}$, and "severe HG" as blood glucose value greater than $200 \mathrm{mg} / \mathrm{dl}$.

All cases were discussed preoperatively in multidisciplinary meeting. All operations were performed with curative intent with classical open approach. After total gastrectomy with D2 lymph node dissection, esophagojejunostomy (using a circular stapler) was used routinely for
Roux-en-Y reconstruction. Proximal and distal resection margins were evaluated intraoperatively to confirm the absence of microscopic disease (R1) in all patients. A Billroth II reconstruction with hand-sewn anastomosis was performed after Subtotal Distal Gastrectomy (including D2 lymph node dissection). Proximal gastrectomy (including D1/D2 lymph node dissection) involved the resection of the proximal half of the stomach via an abdominal approach with intra-abdominal esophagogastric anastomosis using a circular stapler. A Roux-en-Y reconstruction was performed after gastric stump resection as well. In 28 patients with locally advanced gastric cancer, multivisceral resections were performed.

Postoperative complications were considered in a time period of 30 days from surgery and classified according to the classification of Clavien-Dindo [18]. Postoperative mortality was defined as death within 30 days of surgery.

Statistical analysis was performed using descriptive statistics for demographic data. For groupwise comparisons, Student $t$ test (normal distribution) or KruskalWallis test for nonparametric distributions of data were used. To compare nominal data, the $\chi^{2}$ test or the Fisher exact test was used. Multivariate analysis was performed with the logistic regression method. Statistical analysis was performed using commercially available software (SPSS ${ }^{\circledR}$ for Windows version 20.0; Chicago, IL).

\section{Results}

Patients' demographic characteristics and surgical procedures are reported in Table 1.

A total of 193 gastric operations for gastric cancer were performed at our institution during the study period. Of 193 patients, $17(8.8 \%)$ with preexisting diabetes mellitus and $3(1.7 \%)$ on whom pancreatic resections were performed were excluded from the analysis. One hundred and seventy-three patients were nondiabetic and 96 of them $(55.5 \%)$ experienced postoperative $\mathrm{HG}$ of more than $125 \mathrm{mg} / \mathrm{dl}: 85$ patients $(49.1 \%)$ with mild $\mathrm{HG}$ and 11 (6.3\%) with severe HG (Fig. 1).

The most frequent gastric operation was subtotal distal gastrectomy (92 patients), followed by total gastrectomy in 54 patients, proximal gastrectomy in 20 patients, and gastric stump resection in seven patients. Twenty-eight patients $(16.2 \%)$ needed a multivisceral resection because of locally advanced gastric cancer infiltrating adjacent organs. Only 21 patients $(12.1 \%)$ needed preoperative neoadjuvant therapy. Thirty-two patients $(18.5 \%)$ underwent intraoperative blood transfusion.

We compared postoperative outcomes among the three groups, examining postoperative complications, length of stay, perioperative mortality and reoperation rate (Table 1). 
Table 1 Perioperative outcomes

\begin{tabular}{|c|c|c|c|c|c|}
\hline & Overall $(n=173)$ & Normoglicemic $(n=77)$ & Mild HG $(n=85)$ & Severe HG $(n=11)$ & $p$ \\
\hline Length of stay (days; median; range) & 8 & $8(6-37)$ & $8(6-62)$ & $9(6-213)$ & n.s. \\
\hline Complications* & $43(24.8 \%)$ & $10(13 \%)$ & $26(30.6 \%)$ & $7(63.6 \%)$ & \\
\hline Clavien I/II & $25(\%)$ & $6(60 \%)$ & $17(65.4 \%)$ & $2(28.6 \%)$ & $<0.001$ \\
\hline Clavien III/IV & $18(41.8 \%)$ & $4(40 \%)$ & $9(34.6 \%)$ & $5(71.4 \%)$ & \\
\hline Anastomotic leak & $10(5.8 \%)$ & $3(3.9 \%)$ & $5(5.9 \%)$ & $2(18.2 \%)$ & \\
\hline Intrabdominal abscess & $6(3.5 \%)$ & $1(1.3 \%)$ & $3(3.5 \%)$ & $2(18.2 \%)$ & \\
\hline Ileus & $1(0.6 \%)$ & $1(1.3 \%)$ & 0 & 0 & \\
\hline Pneumonia & $15(8.7 \%)$ & $3(3.9 \%)$ & $10(11.7 \%)$ & $2(18.2 \%)$ & \\
\hline Other complications & $15(8.7 \%)$ & $3(3.9 \%)$ & $8(9.4 \%)$ & $4(36.3 \%)$ & \\
\hline Pancreatitis & $1(0.6 \%)$ & $1(1.3 \%)$ & 0 & 0 & \\
\hline Pancreatic fistula & 0 & 0 & 0 & 0 & \\
\hline SSI & $8(4.6 \%)$ & $2(2.6 \%)$ & $3(3.5 \%)$ & $3(27 \%)$ & \\
\hline Reoperation & $5(2.8 \%)$ & $2(2.6 \%)$ & $3(3.5 \%)$ & 0 & n.s. \\
\hline Mortality & $7(4.0 \%)$ & $2(2.6 \%) 20$ & $2(2.3 \%)$ & $3(27.3 \%)$ & $<0.001$ \\
\hline
\end{tabular}

n.s. not significant, SSI surgical site infection

* The total number of patients having complications is less than the sum of patients having individual complications because some patients had more than one complication

Fig. 1 An overview of the study cohort with respect to diabetes status and level of glycemia. Note that glucose levels included in this study had to be obtained the first 72 postoperative hours, but outcome variables pertain to 30 postoperative days. $H G$ indicates hyperglycemia

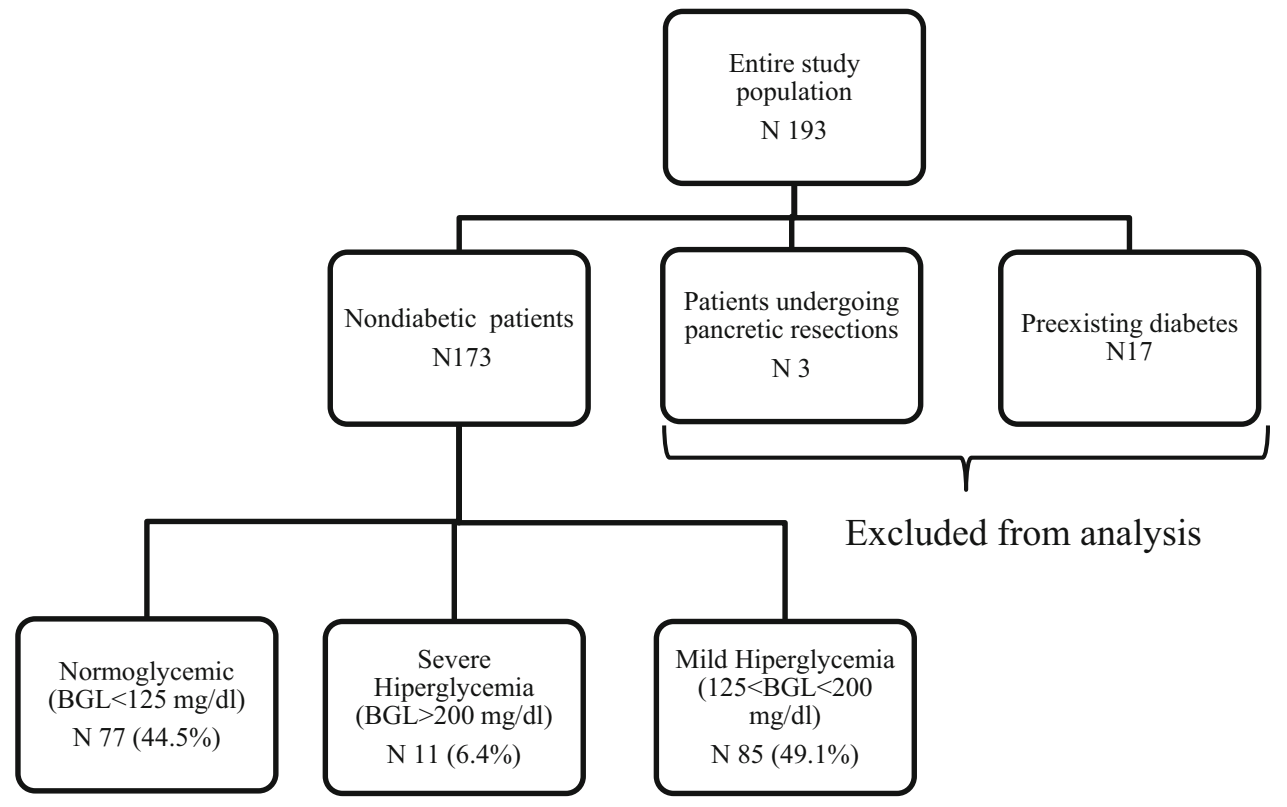

The median postoperative glycemic value for patients with mild HG was $142 \mathrm{mg} / \mathrm{dl}$ (126-193) while in patients with severe HG the median glycemic was $219 \mathrm{mg} / \mathrm{dl}$ (200-494). The mean length of stay was 11.9 days $( \pm 17.8)$. The median postoperative hospital stay was 8 days (4-213). Patients with severe postoperative HG had a longer hospital stay compared to those with mild postoperative HG and normoglycemic patients 9.0 days (6-213), 8 days (6-62) and 8 days (6-37), respectively [ $p$ : not significant (n.s.)].

Total postoperative complication rate was $24.8 \%$ (43 patients out of 173), significantly higher in patients with severe postoperative HG compared to mild HG and normoglycemic patients $(63.6,30.6$, and $13 \%$, respectively, $p<0.001)$. Even if postoperative complications were more frequent in patients with severe HG, no statistical difference was detected among the three groups regarding anastomotic leak incidence. All complications are listed in Table 1.

Comparing demographic and perioperative characteristics with postoperative blood glucose values, only neoadjuvant therapy was related to higher postoperative glucose value. No statistical difference was detected regarding age, 
Table 2 Characteristics of the patients and surgical procedures

\begin{tabular}{|c|c|c|c|c|c|}
\hline & Overall (n. 173) & Normoglycemic ( $n .77)$ & Mild HG (n. 85) & Severe HG $(n .11)$ & $\mathrm{p}$ \\
\hline Age (mean) & 63.89 & 59.09 & 66.59 & 70.13 & n.s. \\
\hline \multicolumn{6}{|l|}{ Gender } \\
\hline Male & $101(58.4 \%)$ & $44(57.1 \%)$ & $49(57.6 \%)$ & $8(72.7 \%)$ & \multirow[t]{2}{*}{ n.s. } \\
\hline Female & $72(41.6 \%)$ & $33(42.9 \%)$ & $36(42.4 \%)$ & $3(27.3 \%)$ & \\
\hline \multicolumn{6}{|l|}{ Type of procedure } \\
\hline Total gastrectomy & $54(31.2 \%)$ & $21(27.3 \%)$ & $28(32.9 \%)$ & $5(45.5 \%)$ & \multirow[t]{4}{*}{ n.s. } \\
\hline Distal gastrectomy & $92(53.2 \%)$ & $45(58.4 \%)$ & $42(49.4 \%)$ & $5(45.5 \%)$ & \\
\hline Proximal gastrectomy & $20(11.6 \%)$ & $9(11.7 \%)$ & $10(11.8 \%)$ & $1(9.1 \%)$ & \\
\hline Gastric stump resection & $7(4.0 \%)$ & $2(2.6 \%)$ & $5(5.9 \%)$ & 0 & \\
\hline \multicolumn{6}{|l|}{ Neoadiuvant therapy } \\
\hline Yes & $21(12.1 \%)$ & $9(11.7 \%)$ & $8(9.4 \%)$ & $4(36.4 \%)$ & \multirow[t]{2}{*}{0.036} \\
\hline No & $152(87.9 \%)$ & $68(88.3 \%)$ & $77(90.6 \%)$ & $7(63.6 \%)$ & \\
\hline \multicolumn{6}{|l|}{ Multivisceral resections* } \\
\hline No & $145(83.8 \%)$ & $67(87 \%)$ & $71(83.3 \%)$ & $7(63.6 \%)$ & \multirow[t]{2}{*}{0.07} \\
\hline Yes & $28(16.2 \%)$ & $10(13 \%)$ & $14(16.7 \%)$ & $4(36.4 \%)$ & \\
\hline \multicolumn{6}{|l|}{ Splenectomy } \\
\hline Yes & $40(23.1 \%)$ & $16(20.8 \%)$ & $19(22.4 \%)$ & $5(45.5 \%)$ & \multirow[t]{2}{*}{ n.s. } \\
\hline No & $133(76.9)$ & $61(79.2 \%)$ & $66(77.6 \%)$ & $6(54.5 \%)$ & \\
\hline \multicolumn{6}{|l|}{ Blood transfusion } \\
\hline Yes & $32(18.5 \%)$ & $12(15.5 \%)$ & $15(17.6 \%)$ & $5(45.5 \%)$ & \multirow[t]{2}{*}{ n.s. } \\
\hline No & $141(81.5 \%)$ & $65(84.5 \%)$ & $70(82.4 \%)$ & $6(54.5 \%)$ & \\
\hline
\end{tabular}

n.s. not significant

* 20 Colon resections, eight liver resections

gender, type of operation, splenectomy and intraoperative blood transfusion (Table 2).

Eighteen out of 28 patients who underwent multivisceral resections showed a slightly higher blood glucose postoperative value, even if this data was not statistically significant $(p=0.07)$. Five patients $(2.8 \%)$ needed reoperation in the postoperative period for intrabdominal sepsis due to anastomotic leak. All of them underwent exploratory laparotomy, peritoneal washing and leak suture. Seven patients $(4.0 \%)$ died in the postoperative period: in all cases due to abdominal sepsis related to anastomotic leak and in one case also due to septic shock. Mortality rate was significantly higher in patients with severe postoperative HG $(27.3 \%)(p<0.001)$ (Table 1$)$.

Additional variables (Age; sex; histopathological features; tumor staging; neoadiuvant therapy; intraoperative blood transfusion; multivisceral resections) were considered, examining perioperative outcomes; results are shown in Tables 3 and 4. Patients affected by advanced tumor had higher rates of perioperative complications (29.7\% stage IIIIV vs. $19.5 \%$ stage I-II; $p=0.03$ ). The need for intraoperative transfusion was associated with higher rates of perioperative mortality (18.7 vs. $0.7 \% p<0.001$ ) (Table 3).
The multivariate analysis showed that severe HG was related to a higher rate of perioperative complications (OR 7.3; IC 1.8-28.4; $p=0.004)$, but not with a higher mortality rate (Table 4$)$.

\section{Discussion}

The glycemic control in the postoperative period is still highly debated in the scientific community, especially because it has never been evaluated in a multicenter study in general surgery patients.

Kwon et al. [19] recently analyzed the relationship between perioperative glycemia and timing of $\mathrm{HG}$, and their impact on postoperative mortality and complications in patients who underwent elective colo-rectal and bariatric surgery, examining a total of 18,278 patients. Blood glycemic value was evaluated the day of surgery, postoperative day one and postoperative day two, defining $\mathrm{HG}$ as blood glucose value $>180 \mathrm{mg} / \mathrm{dl}$. The authors reported that postoperative mortality rate, complication rate and length of hospital stay were significantly higher in patients who experienced HG postoperatively. Significant differences in 

outcomes univariate analysis
Table 3 Factors affecting

\begin{tabular}{|c|c|c|c|c|c|}
\hline & All patients $(n=173)$ & Perioperative complications & $p^{*}$ & Perioperative mortality & $p^{*}$ \\
\hline \multicolumn{6}{|l|}{ TNM } \\
\hline $\mathrm{I} / \mathrm{II}$ & $82(47.4 \%)$ & $16(19.5 \%)$ & 0.03 & $3(3.6 \%)$ & n.s. \\
\hline III/IV & $91(52.6 \%)$ & $27(29.7 \%)$ & & $4(4.4 \%)$ & \\
\hline \multicolumn{6}{|c|}{ Neoadiuvant terapy } \\
\hline Yes & $21(12.1 \%)$ & $7(33.3 \%)$ & n.s. & $1(4.7 \%)$ & n.s. \\
\hline No & $152(87.9 \%)$ & $36(23.7 \%)$ & & $6(3.9 \%)$ & \\
\hline \multicolumn{6}{|c|}{ Multivisceral resections } \\
\hline Yes & $28(16.2 \%)$ & $9(32.1 \%)$ & n.s & $2(7.1 \%)$ & n.s. \\
\hline No & $145(83.8 \%)$ & $34(23.4 \%)$ & & $5(3.4 \%)$ & \\
\hline \multicolumn{6}{|c|}{ Intraoperative blood transfusion } \\
\hline Yes & $32(18.5 \%)$ & $11(34.3 \%)$ & n.s & $6(18.7 \%)$ & $<0.001$ \\
\hline No & $141(81.5 \%)$ & $32(22.7 \%)$ & & $1(0.7 \%)$ & \\
\hline \multicolumn{6}{|l|}{ BGL } \\
\hline$<200$ & $162(93.6 \%)$ & $36(22.2 \%)$ & 0.02 & $4(2.5 \%)$ & $<0.001$ \\
\hline$\geq 200$ & $11(6.4 \%)$ & $7(63.6 \%)$ & & $3(27.2 \%)$ & \\
\hline
\end{tabular}

n.s. not significant

$* \chi$ square or Fisher exact test

Table 4 Factors affecting outcomes multivariate analysis

\begin{tabular}{lll}
\hline & Hazard ratio & $p$ \\
\hline Perioperative complications* & & \\
BGL $(\geq 200)$ & $7.3(1.8-28.4)$ & 0.004 \\
TNM (III/IV) & $3.3(1.5-7.6)$ & 0.003 \\
Perioperative mortality* & & \\
Blood transfusion & $2.7(0.95-7.6)$ & 0.06 \\
BGL $(\geq 200)$ & $6.9(0.9-167.1)$ & 0.05 \\
\hline
\end{tabular}

* Logistic regression

postoperative outcomes were detected in both colorectal and bariatric surgery groups.

Recently, Kiran et al. [13], reported a significant influence of postoperative HG on morbidity and mortality after elective colorectal surgery, demonstrating a higher incidence of medical and surgical complications and mortality in patients with blood glucose values $>125 \mathrm{mg} / \mathrm{dl}$.

We found that $55.5 \%$ of nondiabetic patients who underwent elective gastric surgery experienced high postoperative glycemic values in the first $72 \mathrm{~h}$ after surgery, confirming what was reported by Kwon et al.

Patients with blood glucose values $>126 \mathrm{mg} / \mathrm{dl}$ experienced a significantly higher incidence of postoperative complications $(p=0.004)$. Specifically, 26 out of 85 $(30.6 \%)$ patients with mild $\mathrm{HG}$, and seven out of 11 patients $(63.6 \%)$ with severe $\mathrm{HG}$ had a higher incidence of complications, demonstrating a dose-effect relationship between glycemic control and adverse outcome. Similarly, Kwon et al. [19] demonstrated better postoperative results in patients with postoperative glucose levels lower than
$130 \mathrm{mg} / \mathrm{dl}$ and worse outcomes in patients with postoperative glucose values $>180 \mathrm{mg} / \mathrm{dl}$.

Because of the small size of our study population, it was not possible to analyze the relationship between every single complication and the study population, even if a higher incidence of anastomotic leak and intrabdominal abscess was reported in patients with severe HG (Table 2).

Nondiabetic patients with $\mathrm{HG}$ also had a significantly higher incidence of medical adverse outcomes $(p<0.001)$ and postoperative mortality ( $p<0.001)$. Similarly, Kiran et al. [13] reported a higher incidence of postoperative complication, length of stay and mortality in nondiabetic patients who experienced postoperative $\mathrm{HG}$ after elective colorectal surgery.

One of the strengths of our study is that we focused on a single center population, minimizing potential bias by using the same postoperative management and surgical techniques. We also included in the analysis patients with mild postoperative glycemic value, usually considered unproblematic patients. The results for patients with mild HG are in accordance with those reported in previous studies regarding postoperative outcomes in patients who underwent elective colorectal surgery [18, 20-24].

As reported by Know et al. [19], despite the importance of postoperative $\mathrm{HG}$ and its impact on postoperative outcomes reported in the literature, only $26 \%$ of all patients with $\mathrm{HG}$ (46\% of them were nondiabetic) on the day of surgery did not receive insulin. This may be due to the limited awareness of the importance of perioperative $\mathrm{HG}$ on postoperative outcomes, especially in general surgery [19].

Cao et al., in a single-centre, prospective, randomized controlled trial, compared the effects of intensive insulin 
therapy with conventional insulin therapy on postoperative outcomes among nondiabetic patients receiving parenteral nutrition following D2 gastrectomy for gastric cancer [25]. The authors demonstrated that intensive insulin therapy significantly reduced the postoperative short-term morbidity but not mortality among nondiabetic patients receiving parenteral nutrition after D2 gastrectomy. The benefits may be due to the suppression of insulin resistance and improvement of HLA-DR expression on monocytes [25].

Wang et al. [26], in a prospective study on 255 consecutive patients with gastric cancer, evaluated the association of sarcopenia, characterized by decreased skeletal muscle plus low muscle strength and/or physical performance, with short-term postoperative outcomes after gastrectomy for gastric cancer. At multivariable analysis, this study revealed that sarcopenia and diabetes were independent predictors of postoperative complications [26].

As reported by Kao et al. [27], in a review study published in 2013, stress HG is a risk factor for SSI with cause-effect relationship, on the other hand, there is low quality evidence that treatment of HG with insulin could reduce this risk.

Even with the limitations of its retrospective nature and the small study size, our results are in accordance with the literature, in which a strong relationship between postoperative HG and postoperative outcomes in colorectal and bariatric surgery was found. For these reasons, we might suggest that stress HG could be a risk factor of worse outcomes in gastric surgery for cancer.

More attention should be focused on nondiabetic patients after gastric surgery, with appropriate intervention and monitoring, to reduce and prevent adverse postoperative events as much as possible, especially in patients with severe HG.

Acknowledgments The funders had no role in study design, data collection and analysis, decision to publish, or preparation of the manuscript.

\section{Compliance with ethical standards}

Conflict of interest The authors declare that they have no conflict of interest.

Human rights statement and informed consent All procedures followed were in accordance with the ethical standards of the responsible committee on human experimentation (institutional and national) and with the Helsinki Declaration of 1964 and later versions. Informed consent or substitute for it was obtained from all patients for being included in the study.

\section{References}

1. McAlister FA, Man J, Bistritz L, et al. Diabetes and coronary artery bypass surgery: an examination of perioperative glycemic control and outcomes. Diabetes Care. 2003;26:1518-24.

2. Latham R, Lancaster AD, Covington JF, et al. The association of diabetes and glucose control with surgical-site infections among cardiothoracic surgery patients. Infect Control Hosp Epidemiol. 2001;22:607-12.

3. McGirt MJ, Woodworth GF, Brooke BS, et al. Hyperglycemia independently increases the risk of perioperative stroke, myocardial infarction, and death after carotid endarterectomy. Neurosurgery. 2006;58:1066-73.

4. Park C, Hsu C, Neelakanta G, et al. Severe intraoperative hyperglycemia is independently associated with surgical site infection after liver transplantation. Transplantation. 2009;87:1031-6.

5. Ambiru S, Kato A, Kimura F, et al. Poor postoperative blood glucose control increases surgical site infections after surgery for hepato-biliary-pancreatic cancer: a prospective study in a highvolume institute in Japan. J Hosp Infect. 2008;68:230-3.

6. McConnell YJ, Johnson PM, Porter GA. Surgical site infections following colorectal surgery in patients with diabetes: association with postoperative hyperglycemia. J Gastrointest Surg. 2009;13:508-15.

7. van den Berghe $G$, Wouters $P$, Weekers $F$, et al. Intensive insulin therapy in the critically ill patients. $N$ Engl $J$ Med. 2001;345:1359-67.

8. Finney SJ, Zekveld C, Elia A, et al. Glucose control and mortality in critically ill patients. JAMA. 2003;290:2041-7.

9. Serra-Aracil X, Garcia-Domingo MI, Pares D, et al. Surgical site infection in elective operations for colorectal cancer after the application of preventive measures. Arch Surg. 2011;146:606-12.

10. Turina M, Miller FN, Tucker CF, et al. Short-term hyperglycemia in surgical patients and a study of related cellular mechanisms. Ann Surg. 2006;243:845-51.

11. Ata A, Lee J, Bestle SL, et al. Postoperative hyperglycemia and surgical site infection in general surgery patients. Arch Surg. 2010;145:858-64.

12. Ho VP, Stein SL, Trencheva K, et al. Differing risk factors for incisional and organ/space surgical site infections following abdominal colorectal surgery. Dis Colon Rectum. 2011;54:818-25.

13. Kiran RP, Turina M, Hammel J, Fazio V. The clinical significance of an elevated postoperative glucose value in non-diabetic patients after colorectal surgery: evidence for the need for tight glucose control? Ann Surg. 2013;258:599-604.

14. Levetan CS, Passaro M, Jablonski K, et al. Unrecognized diabetes among hospitalized patients. Diabetes Care. 1998;21:246-9.

15. Furnary AP, Gao G, Grunkemeier GL, et al. Continuous insulin infusion reduces mortality in patients with diabetes undergoing coronary artery bypass grafting. J Thorac Cardiovasc Surg. 2003; 125:1007-21.

16. Furnary AP, Zerr KJ, Grunkemeier GL, et al. Continuous intravenous insulin infusion reduces the incidence of deep sternal wound infection in diabetic patients after cardiac surgical procedures. Ann Thorac Surg. 1999;67:352-60.

17. Rosa F, Alfieri S, Tortorelli AP, Fiorillo C, Costamagna G, Doglietto GB. Trends in clinical features, postoperative outcomes, and long-term survival for gastric cancer: a Western experience with 1278 patients over 30 years. World J Surg Oncol. 2014;12:217.

18. Dindo D, Demartines N, Clavien P-A. Classification of surgical complications a new proposal with evaluation in a cohort of 6336 patients and results of a survey. Ann Surg. 2004;240(2):205-13.

19. Kwon S, Thompson S, Dellinger P, et al. Importance of perioperative glycemic control in general surgery: a report from the surgical care and outcomes assessment program. Ann Surg. 2013;257:8-14.

20. Schmeltz LR, DeSantis AJ, Thiyagarajan V, et al. Reduction of surgical mortality and morbidity in diabetic patients undergoing cardiac surgery with a combined intravenous and subcutaneous insulin glucose management strategy. Diabetes Care. 2007;30:823-8. 
21. Bochicchio GV, Sung J, Joshi M, et al. Persistent hyperglycemia is predictive of outcome in critically ill trauma patients. J Trauma. 2005;58:921-4.

22. Laird AM, Miller PR, Kilgo PD, et al. Relationship of early hyperglycemia to mortality in trauma patients. J Trauma. 2004;56:1058-62.

23. Griesdale DE, de Souza RJ, van Dam RM, et al. Intensive insulin therapy and mortality among critically ill patients: a meta-analysis including NICESUGAR study data. CMAJ. 2009;180:821-7.

24. Jackson RS, Amdur RL, White JC, et al. Hyperglycemia is associated with increased risk of morbidity and mortality after colectomy for cancer. J Am Coll Surg. 2012;214:68-80.
25. Cao $\mathrm{S}$, Zhou $\mathrm{Y}$, Chen $\mathrm{D}$, et al. Intensive versus conventional insulin therapy in nondiabetic patients receiving parenteral nutrition after D2 gastrectomy for gastric cancer: a randomized controlled trial. J Gastrointest Surg. 2011;15:1961-8.

26. Wang SL, Zhuang CL, Huang DD, et al. Sarcopenia adversely impacts postoperative clinical outcomes following gastrectomy in patients with gastric cancer: a prospective study. Ann Surg Oncol. 2016;23:556-64.

27. Kao LS, Phatak UR. Glycemic control and prevention of surgical site infection. Surgical Infections. 2013;14(5):437-44. 